OPEN ACCESS

Edited by:

Jie-Sheng Chen,

Shanghai Jiao Tong University, China

Reviewed by:

Jie Shu,

Ningbo University, China Yonggang Wang

Fudan University, China

Mingdeng Wei,

Fuzhou University, China

*Correspondence:

Chunhai Jiang

chiiang@xmut.edu.cn

Specialty section

This article was submitted to

Colloidal Materials and Interfaces, a section of the journal

Frontiers in Materials

Received: 28 August 2018 Accepted: 20 September 2018

Published: 10 October 2018

Citation:

Zou Z, Wu L, Chen J and Jiang C (2018) A Sandwich-Structured Hybrid

Anode With Nitrogen-Doped Amorphous Carbon Nanoarrays Vertically Anchoring on Graphene

Nanoplatelets for High Rate L

Storage. Front. Mater. 5:61.

doi: 10.3389/fmats.2018.00061

\section{A Sandwich-Structured Hybrid Anode With Nitrogen-Doped Amorphous Carbon Nanoarrays Vertically Anchoring on Graphene Nanoplatelets for High Rate Li Storage}

\author{
Zhimin Zou, Limin Wu, Jianwei Chen and Chunhai Jiang* \\ Fujian Provincial Key Laboratory of Functional Materials and Applications, School of Materials Science and Engineering, \\ Institute of Advanced Energy Materials, Xiamen University of Technology, Xiamen, China
}

Graphene is not an ideal anode material of Li-ion batteries because of its low packing density and low initial Coulombic Efficiency although it shows much higher specific capacity than graphite. Herein, we report a sandwich-structured hybrid anode material which integrates the nitrogen-doped amorphous carbon nanoarrays on both sides of graphene nanoplatelets. The former provides high capacity and excellent rate capability, while the latter stabilizes the cycle performance, both of them brought out outstanding electrochemical properties to the hybrid anode. High discharge capacities of 562 and $217 \mathrm{~mA} \mathrm{~h} \mathrm{~g}^{-1}$ are obtained at current densities of 0.1 and $3 \mathrm{~A} \mathrm{~g}^{-1}$, respectively, which are much higher than those of the starting graphene nanoplatelets (404 and $81 \mathrm{~mA} \mathrm{~h}$ $\mathrm{g}^{-1}$, respectively). Moreover, a discharge capacity of $540 \mathrm{~mA} \mathrm{~h} \mathrm{~g}^{-1}$ is maintained after 300 cycles at $0.5 \mathrm{~A} \mathrm{~g}^{-1}$, demonstrating an excellent cycle stability. This study provides a facile process to prop up the $2 \mathrm{D}$ graphene nanoplatelets with vertically aligned carbon nanoarrays, which may push forward the application of graphene as anode material of $\mathrm{Li}$-ion batteries because of the avoided aggregation and additional Li storage capacity contributed by the $\mathrm{N}$-doped amorphous carbon.

Keywords: Li-ion batteries, graphene nanoplatelets, anode, composite, rate capability

\section{INTRODUCTION}

The rapid development of electric vehicles (EVs) calls for reliable energy storage devices with both high energy and high power densities. Li-ion batteries (LIBs) are currently the most widely used power sources of EVs, but their low energy density is still one major concern of EV manufacturers and customers, as one always hopes to drive as long as possible at a single charge. The inferior energy and power densities of LIBs are majorly determined by the intrinsic characteristics of their electrode materials (Nitta et al., 2015). Taking the state-of-theart anode material of LIBs, graphite, as an example, it has high reversibility and stable cycle performance, but limited capacity (only $372 \mathrm{~mA} \mathrm{~h} \mathrm{~g}^{-1}$ theoretically) and rate performance. This makes graphite not an ideal anode material for high power LIBs (Zhang et al., 2014). 
The low capacity and poor rate performance of graphite are ascribed to its low number of active sites and narrow lattice space ( $~ 0.335 \mathrm{~nm}$; Striebel et al., 2004). Reducing the thickness of graphite is found effective to improve its specific capacity and rate performance because of the introduction of additional $\mathrm{Li}$-ion storage sites and shortening of the Li-ion diffusion distance. For an instance, ultrathin layered graphite prepared by jet impinging apparatus showed a high reversible capacity of $393 \mathrm{~mA} \mathrm{~h} \mathrm{~g}^{-1}$, obviously higher than the $345 \mathrm{~mA} \mathrm{~h} \mathrm{~g}^{-1}$ of traditional graphite (Chen et al., 2018). As one-atom layer or few-atom layers ultrathin two-dimensional graphite, graphene has shown further increased lithium storage capacity in comparison to ultrathin layered graphite. Specific capacities of 1,175, 1,007, and $842 \mathrm{~mA} \mathrm{~h}$ $\mathrm{g}^{-1}$ have been reported on graphene sheets with single, triple and quintuplicate layers, respectively (Tong et al., 2011). However, graphene is not a suitable anode material of commercial LIBs because of its low packing density, large irreversible capacity loss in the first cycle and the capacity fluctuation related to the complex surface chemistry and electrochemical reactions at the electrolyte/electrode interface (Dong et al., 2017). Instead, hybridizing graphene with other high capacity active materials, such as silicon (Assresahegn et al., 2018), metal oxides (Liu et al., 2015; Qiu et al., 2015; Hong et al., 2016; Jiang et al., 2018; Shi et al., 2018; Wang et al., 2018; Zhang et al., 2018), and metal sulfides (Che et al., 2016; Wang et al., 2017), etc., has been the frequently used strategy to develop high performance anode materials.

Besides graphene, amorphous carbons have been recognized as another family of high capacity anode materials of LIBs with specific capacity up to $1,000 \mathrm{~mA} \mathrm{~h}^{-1}$ (Qie et al., 2012; Li et al., 2013; Dutta et al., 2014). Such a high capacity is ascribed to the additional lithium storage at the interface or defects in amorphous carbons. The small crystalline size and wider interlayer distance may have also speed up the $\mathrm{Li}^{+}$ ions diffusion within the disordered structure of amorphous carbons, resulting in outstanding rate capability. However, in most studies, amorphous carbon anodes suffer from low initial Coulombic Efficiencies and poor cycle stability due to pulverization of the carbon particles, variations in surface morphology, disordered non-crystallized sites, random lattice structures and contamination from metallic impurities (Wu et al., 1999; Fujimoto, 2010). The severe polarization of amorphous carbon anodes also prevents them from practical applications in LIBs.

Taking the advantages of high stability of graphite and high capacity of amorphous carbons, hybridizing them may yield potential high performance anode materials with both high capacity and high cycle stability. However, such works are so far mainly carried out on graphene or reduced graphene oxide (rGO) substrates. Zhou et al fabricated a three-dimensional carbon-coated graphene anode, which exhibited excellent Nastorage performance, with a high initial discharge capacity of $824 \mathrm{~mA} \mathrm{~h} \mathrm{~g}^{-1}$ at $50 \mathrm{~mA} \mathrm{~g}^{-1}$ (Zhou et al., 2017). Liu et al prepared a nitrogen-doped carbon/graphene hybrid anode for Na-ion batteries, which showed an excellent rate performance, namely $94 \mathrm{~mA} \mathrm{~h} \mathrm{~g}^{-1}$ at $5 \mathrm{~A} \mathrm{~g}^{-1}$ (Liu et al., 2016). Xu and coauthors fabricated a hybrid anode with vertically aligned carbon nanotubes growing on few-layer graphene. The obtained electrode exhibited a reversible Li storage capacity of $525 \mathrm{~mA} \mathrm{~h}$ $\mathrm{g}^{-1}$ after 1,000 cycles at $0.5 \mathrm{~A} \mathrm{~g}^{-1}$ with Coulombic Efficiency inexcess of $98 \%$ (Xu et al., 2018). To date, hybrid anodes based on graphene nanoplatelets and amorphous carbon are rarely reported although the former exhibited more stability and higher packing density than graphene and rGO.

In this work, a sandwich-structured hybrid anode with nitrogen-doped amorphous carbon nanoarrays vertically anchoring on graphene nanoplatelets was prepared by a $\mathrm{MnO}_{2}$ templated polymerization and post-annealing process (Jiang et al., 2017). The hybrid anode displayed obvious higher specific capacity and rate capability than the starting graphene nanoplatelets for Li storage. The detailed preparation, characterizations and electrochemical performance of the hybrid anode are reported in this paper.

\section{EXPERIMENTAL}

\section{Sample Preparation and Characterizations}

The chemicals used in this work are analytical grade and used as received. The graphene nanoplatelets (about 6-10 layers) were purchased from Suzhou Tanfeng Graphene Sci. and Tech. Co. Ltd, China. The sandwich-structured hybrid anode material was prepared as follows: $0.05 \mathrm{~g}$ graphene nanoplatelets were dispersed in $200 \mathrm{ml}$ ultrapure water holding in a $300 \mathrm{ml}$ glass beaker under magnetic stirring. $0.3 \mathrm{~g} \mathrm{KMnO}_{4}$ (Aladdin Chemistry) and $0.6 \mathrm{ml}$ concentrated $\mathrm{HCl}$ were added into the beaker in sequence under stirring. The beaker was then sealed tightly by a piece of thin polyethylene film and put into an oil bath holding at $80^{\circ} \mathrm{C}$. The magnetic stirring was maintained for another few hours till the purple color of the solution disappeared. With this treatment, vertically aligned birnessite $\mathrm{MnO}_{2}$ nanosheets were coated on both sides of the graphene nanoplatelets. After filtering and washing with ultrapure water a few times, the product was re-dispersed in $100 \mathrm{ml} 0.5 \mathrm{M} \mathrm{HCl}$ solution under magnetic stirring. Then, $0.3 \mathrm{ml}$ pyrrole monomer (Aladdin Chemistry) was dropped into the solution slowly. After being stirred for another $3 \mathrm{~h}$, the product was filtered out, washed repeatedly with ultrapure water and dried at $80^{\circ} \mathrm{C}$. By this process, the birnessite $\mathrm{MnO}_{2}$ nanosheets were in situ replaced by polypyrrole (PPy) nanoasheets via a redox polymerization reaction. The PPy nanoasheets were finally converted to nitrogen-doped amorphous carbon (NC) nanoarrays by annealing at $500^{\circ} \mathrm{C}$ for $2 \mathrm{~h}$ in flowing nitrogen. To simplify the description, the graphene nanoplatelets and graphene nanoplatelets coated by $\mathrm{N}$-doped amorphous carbon nanoarrays were named as G and GNC, respectively.

The graphene nanoplatelets and resulting samples were characterized by powder X-ray diffraction (XRD, Rigaku MiniFlex600), X-ray photoelectron spectroscopy (XPS, ESCALAB250, Thermo VG), Raman Spectrometer (RM1000, Renishaw), scanning electronic microscope (SEM, Zeiss Sigma 500), and transmission electronic microscope (TEM, FEI Talos F200s), respectively.

\section{Electrochemical Measurements}

Electrochemical performances of the graphene nanoplatelets and the sandwich-structured hybrid anode material were evaluated by coin-type Li-metal half cells. The working electrodes were 


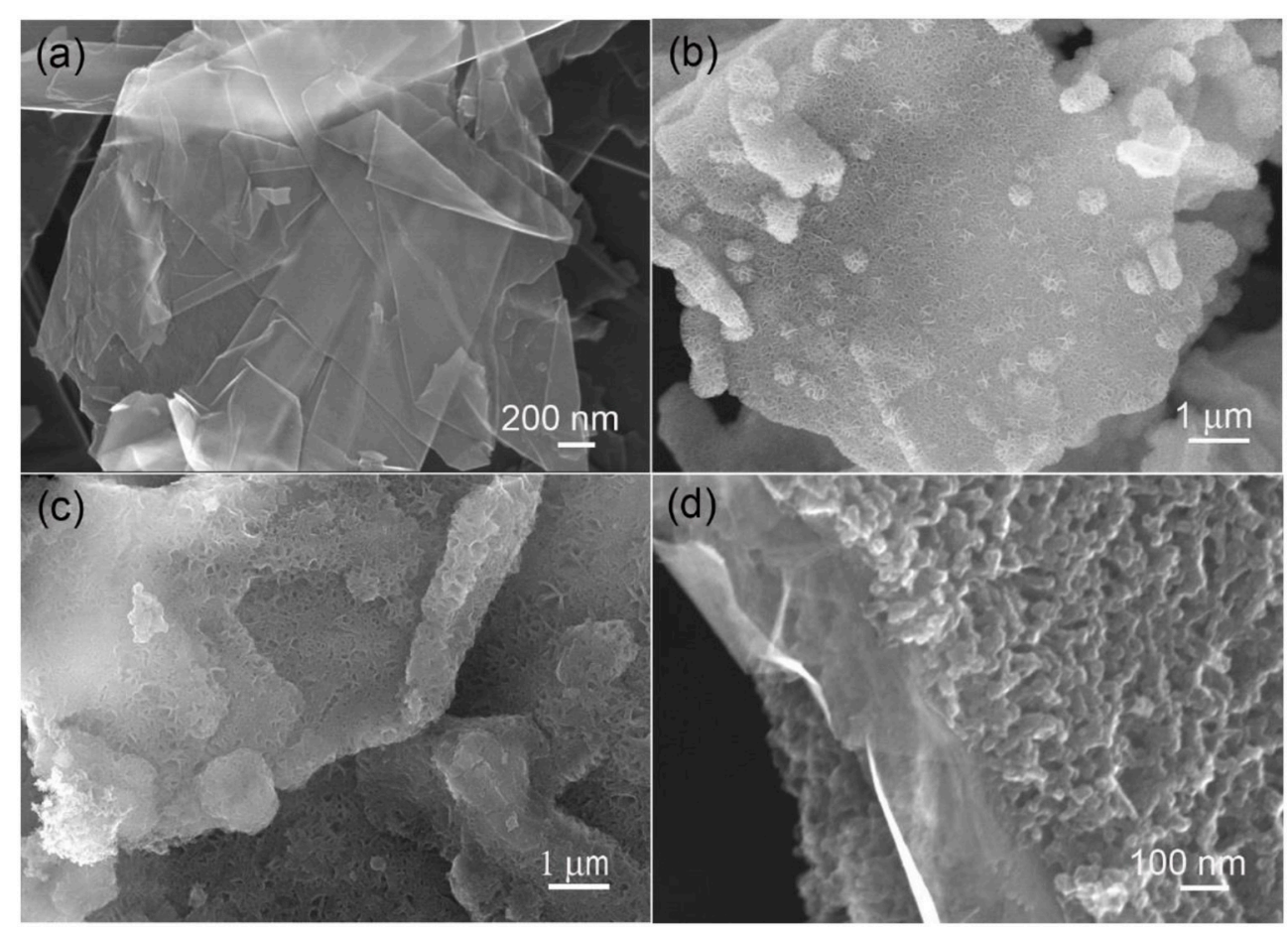

FIGURE 1 | SEM images of the starting graphene nanoplatelets (a), the graphene nanoplatelets coated by birnessite $\mathrm{MnO}_{2}$ (b), PPy (c), and $\mathrm{N}$-doped amorphous carbon nanoarrays (d).

prepared by mixing $80 \mathrm{w} \%$ of the active materials ( $\mathrm{G}$ and GNC), $10 \mathrm{wt} \%$ of acetylene carbon black and $10 \mathrm{wt} \%$ of polyvinylidene fluoride binder in N, N-dimethyl pyrrolidone (NMP) solvent and then coated on copper foil by a doctor-blade method. The loading mass of active materials in each electrode was about $0.8-1.0 \mathrm{mg}$ $\mathrm{cm}^{-2}$. The electrolyte was a $1 \mathrm{M}$ solution of $\mathrm{LiPF}_{6}$ dissolved in ethylene carbonate (EC) and dimethyl carbonate (DEC) with a volume ratio of 1:1. The 2025-type coin cells were assembled in an argon filled glove box with lithium metal as the counter electrode and polypropylene membrane (Celgard 2400) as the separator, respectively. Galvonostatic discharge/charge measurements were performed in the voltage range of $0.01-3.0 \mathrm{~V}$ (vs. $\mathrm{Li} / \mathrm{Li}^{+}$) using a battery cycler (LAND CT2001A, Wuhan Kingnuo Electronic Co., China). The cyclic voltammetry curves were recorded in the voltage range of $0.01-3.0 \mathrm{~V}$ (vs. $\mathrm{Li} / \mathrm{Li}^{+}$) on a $\mathrm{CHI} 600 \mathrm{e}$ electrochemical workstation (Chenhua, Shanghai, China) at a scan rate of $0.1 \mathrm{mV} \mathrm{s}^{-1}$. Electrochemical impedance spectra (EIS) were measured on the same electrochemical workstation in a frequency range of $10 \mathrm{MHz}$ to $0.01 \mathrm{~Hz}$ with an alternative amplitude of $10 \mathrm{mV}$.

\section{RESULTS AND DISCUSSION}

The microstructures of the starting graphene nanoplatelets and their composites with $\mathrm{MnO}_{2}$, PPy and $\mathrm{N}$-doped amorphous carbon were examined by SEM at first, as shown in Figure 1. From Figure 1a it is seen that the starting graphene nanoplatelets are composed of stacked ultrathin layered graphite with planar size from a few to several tens of micrometer squares. After a soft hydrothermal treatment with $\mathrm{KMnO}_{4}$ solution, the graphene nanoplatelets were uniformly coated by vertically aligned $\mathrm{MnO}_{2}$ nanosheets on both sides (Figure 1b). By an in situ polymerization process in acidic solution, the $\mathrm{MnO}_{2}$ nanosheets were replaced by polypyrrole nanosheets (Figure 1c). By a low temperature heat treatment at $500^{\circ} \mathrm{C}$ for $2 \mathrm{~h}$ in flowing nitrogen, the PPy nanosheets were converted to $\mathrm{N}$-doped amorphous carbon nanoarrasys, forming a sandwich-structured hybrid material (Figure 1d).

The microstructure of the sandwich-structured hybrid material was further characterized by TEM analysis, as shown in Figure 2. The bright-field TEM images shown in Figures 2a,b at different magnifications indicate that the amorphous carbon nanoarrays vertically grew on both sides of the graphene nanoplatelets. The typical thickness of the sandwich structure is around $300 \mathrm{~nm}$. A HADDF image (Figure 2c) clearly reveals that the carbon nanoarrays growing on the thin graphene nanoplatelets are highly porous. The $\mathrm{C}$ (Figure 2d) and $\mathrm{N}$ (Figure 2e) element maps of the hybrid material corresponding to image shown in Figure $2 \mathrm{c}$ demonstrate that the carbon coating layers derived from PPy nanosheets are uniformly doped by nitrogen. This is consistent to the results of our previous publication, i.e., PPy can be converted to $\mathrm{N}$-doped amorphous carbon by low temperature annealing in inert atmosphere (Jiang et al., 2017).

The XRD patterns of the starting graphene nanoplatelets and their composites with $\mathrm{MnO}_{2}$ and $\mathrm{N}$-doped carbon nanosheests 


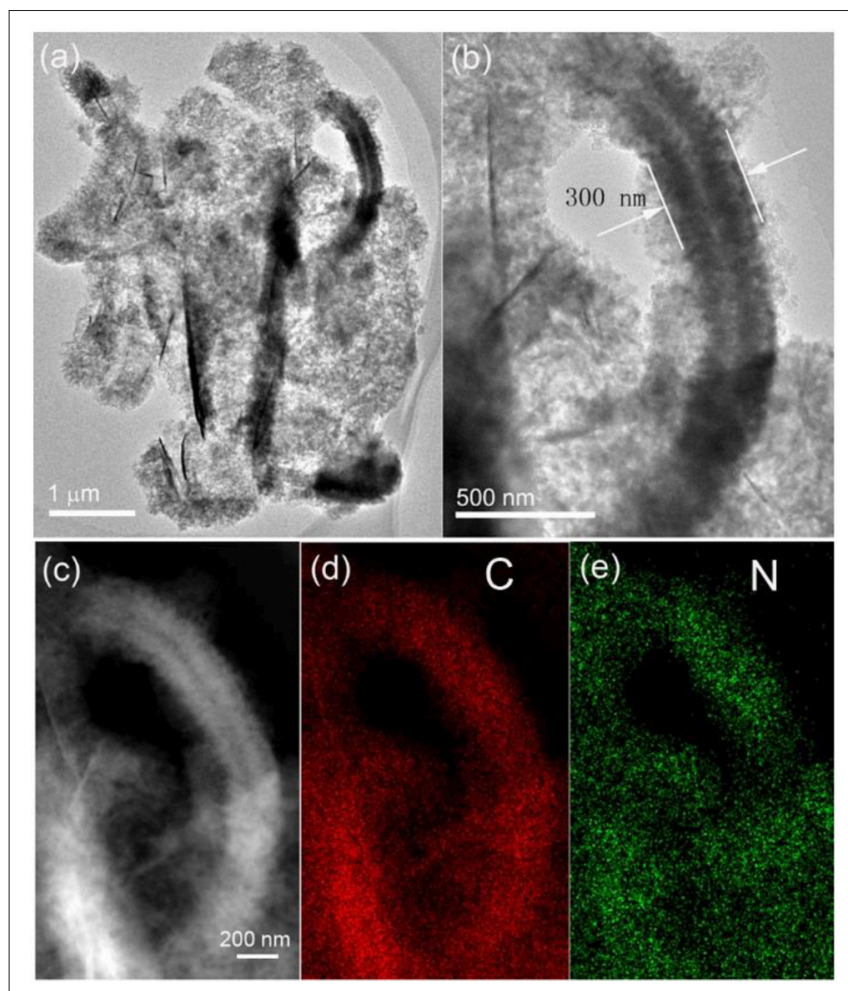

FIGURE 2 | Bright-field TEM images (a,b), HADDF image (c) of the sandwich-structured hybrid material, and the element maps of $\mathrm{C}$ (d), and $\mathrm{N}$ (e) corresponding to the figure shown in (c).

are displayed in Figure 3A. The XRD pattern of the starting graphene nanoplatelets show the typical $\left(\begin{array}{lll}0 & 0 & 2\end{array}\right)$ diffraction peak of graphite at $2 \theta=26.4^{\circ}$, indicating that the starting materials are multilayer graphene. After hydrothermal treatment with $\mathrm{KMnO}_{4}$, only weak $\left(\begin{array}{lll}0 & 0 & 2\end{array}\right)$ diffraction peak of graphite is detected. The sample is fully covered with birnessite $\mathrm{MnO}_{2}$. In the XRD pattern of GNC, except the strong peak of $\left(\begin{array}{lll}0 & 0 & 2\end{array}\right)$, there is also a broad diffraction peak at around $2 \theta=26^{\circ}$, indicating the amorphous nature of the obtained $\mathrm{N}$-doped carbon nanoarrays. This result is consistent to our previous report that annealing PPy at a low temperature would result in $\mathrm{N}$-doped amorphous carbon with low graphitization degree (Jiang et al., 2017).

The starting graphene nanoplatelets and GNC sample were further characterized by Raman spectroscopy (Figure 3B). The starting graphene nanoplatelets have a weak D band at 1,333 $\mathrm{cm}^{-1}$, a sharp $\mathrm{G}$ band at $1,580 \mathrm{~cm}^{-1}$ and a broad $2 \mathrm{D}$ band at $2,680 \mathrm{~cm}^{-1}$. These characteristic peaks are similar to that of bulk graphite (Liu et al., 2017). This again indicates that the starting materials are multilayer graphene. The GNC shows an overlapped Raman spectrum of the graphene nanoplatelets and the $\mathrm{N}$-doped amorphous carbon produced from PPy. The increased intensity of D band in GNC is related to the disorder introduced by defects or heteroatoms, which further confirms nitrogen doping in the amorous carbon nanoarrays. Figures 3C,D show the XPS C1s and N1s core scans of the GNC. As can be seen in Figure 3C, the C1s XPS spectrum can be deconvoluted into five different peaks at 284.0, 284.6, 285.2, 286.2, and $289.2 \mathrm{eV}$, respectively. According to Selvamani et al, $284.0 \mathrm{eV}$ represents the $\mathrm{sp}^{3} \mathrm{C}, 284.6 \mathrm{eV}$ corresponds to the $\mathrm{sp}^{2}$ $\mathrm{C}=\mathrm{C}$, and $285.2 \mathrm{eV}$ is related to the combination of $\mathrm{sp}^{3} \mathrm{C}$ and $\mathrm{C}$ $\mathrm{N}$ bonding. The $286.2 \mathrm{eV}$ belongs to the pyridinic carbon linked to nitrogen $\mathrm{C}=\mathrm{N} / \mathrm{C}=\mathrm{O}$, and $289.2 \mathrm{eV}$ is associated with $\mathrm{O}-\mathrm{C}=\mathrm{O}$ bonding (Selvamani et al., 2016). The N1s spectrum can also be deconvoluted into pyrrolic $\mathrm{N}$ and pyridicnic N (see Figure 3D), similar to the results reported in our previous publication (Jiang et al., 2017). In combination with the $\mathrm{N}$ clement map in Figure 2e, it is evident that the carbon nanoarrays anchoring on the graphene nanoplatelets are uniformly doped by nitrogen.

The electrochemical performances of the starting graphene nanoplatelets and GNC were firstly evaluated by $\mathrm{CV}$ measurements at $0.1 \mathrm{mV} \mathrm{s}^{-1}$ within a voltage range of $0.01-3 \mathrm{~V}$ (vs. $\mathrm{Li} / \mathrm{Li}^{+}$), as shown in Figure 4A. The electrode made of graphene nanoplatelets exhibits a typical CV curve of graphite, which includes a reduction peak at around $0.67 \mathrm{~V}$ in the first cathodic scan, corresponding to the formation of Solid Electrolyte Interphase (SEI) film, and a pair of sharp redox peaks at $0.01 \mathrm{~V} / 0.25 \mathrm{~V}$ in the following cathodic and anodic scan due to the insertion/extraction of $\mathrm{Li}^{+}$ions into/from graphite interlayer lattice (Wang and Yoshio, 2001). The redox peaks at about 0.16 $\mathrm{V} / 0.28 \mathrm{~V}$ appearing from the second cycle also correspond to lithiation and delithiation processes. The GNC electrode exhibits left shift of the cathodic peak in the first scan and increased voltage gap between the cathodic and anodic scans as compared to that of the graphene nanoplatelets. This should be ascribed to the overlap of $\mathrm{CV}$ curves of the $\mathrm{N}$-doped amorphous carbon and the graphene nanoplatelets. The $\mathrm{N}$-doped amorphous carbon has lower $\mathrm{Li}^{+}$intercalation potential than graphite, which then has lowered the SEI formation potential. The peak at about $1.38 \mathrm{~V}$ in the anodic scan can be assigned to the reaction of $\mathrm{Li}^{+}$ions with $\mathrm{N}$-containing functional groups (Jiang et al., 2017). The larger area of CV loop of the GNC electrode suggests that it has higher Li storage capacity.

The rate capability of the G and GNC electrodes are shown in Figure 4B. It is clear to see that, at all current densities, GNC exhibits higher specific capacity than the graphene nanoplatelets. For example, the average discharge capacities of GNC at 0.1 (except the first cycle), $0.2,0.5,1,2$, and $3 \mathrm{~A} \mathrm{~g}^{-1}$ are about 562, $482,398,318,242$, and $217 \mathrm{~mA} \mathrm{~h} \mathrm{~g}^{-1}$, respectively, whereas the corresponding values for the graphene nanoplatelets are 404, $372,324,252,135$, and $81 \mathrm{~mA} \mathrm{~h} \mathrm{~g}^{-1}$, respectively. This indicates that hybridizing $\mathrm{N}$-doped amorphous carbon nanoarrays with graphene nanoplatelets has greatly enhanced the lithium storage capacity of the latter. This is reasonable as the PPy-derived $\mathrm{N}$-doped amorphous carbon possess not only higher capacity but better rate capability than graphite due to the nitrogen doping induced enhancement of electronic conductivity and better wetting of the electrode by electrolyte. The vertically aligned $\mathrm{N}$-doped amorphous carbon nanoarrays might have also facilitated the $\mathrm{Li}^{+}$ions transportation within the electrode material, resulting in much improved rate capability. From Figure 4B we can also see that, the Coulombic Efficiencies for GNC and the starting graphene nanoplatelets at the first cycle are 60.3 and $72.6 \%$, respectively. The lowered initial Coulombic 


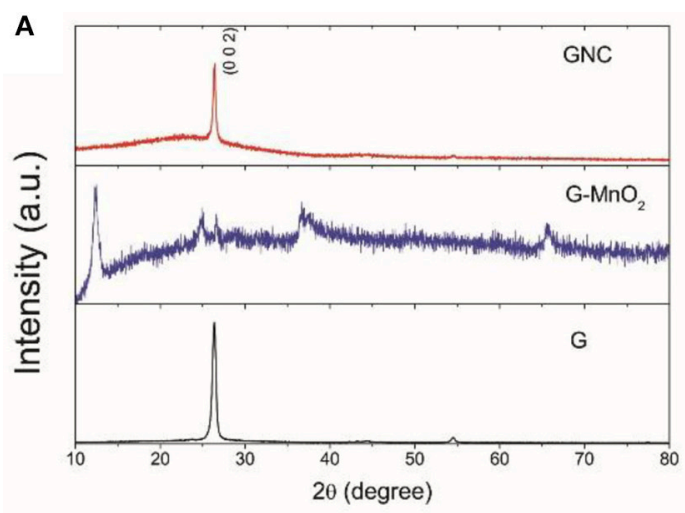

C

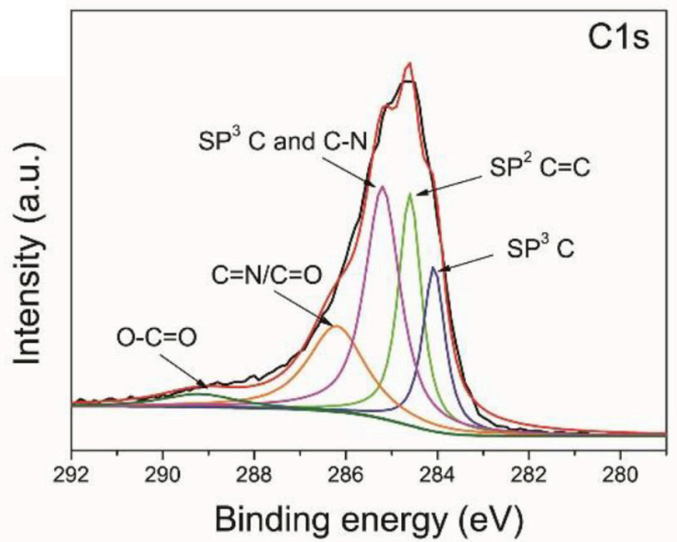

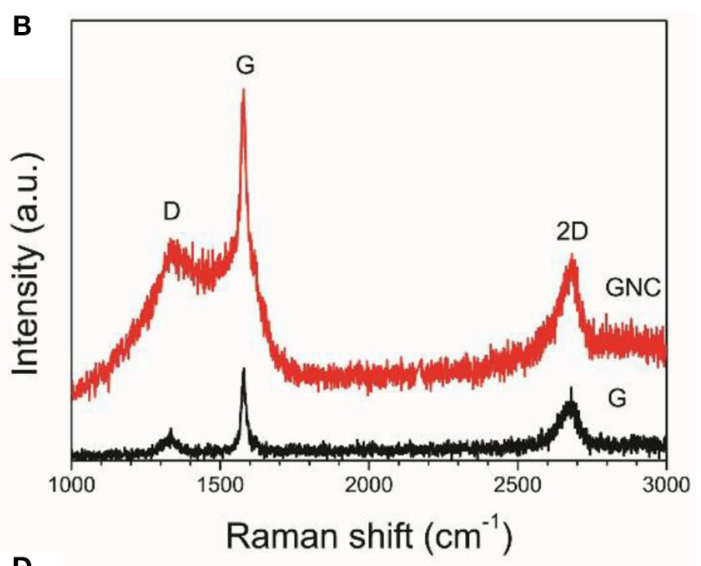

D

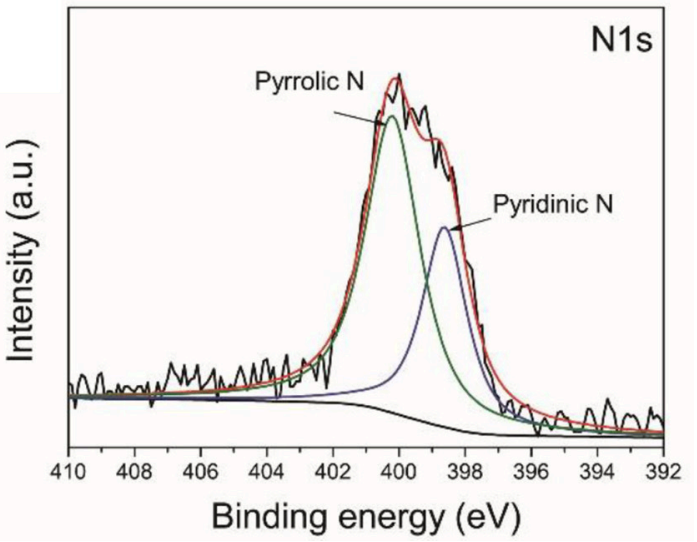

FIGURE 3 | XRD patterns of the starting graphene nanoplatelets and their composite with birnessite $\mathrm{MnO}_{2}$ and $\mathrm{N}$-doped amorphous carbon (A), the Raman spectra of the starting graphene nanoplatelets and GNC (B), the XPS C1s (C), and N 1s (D) core scans of GNC.

Efficiency can be ascribed to the larger irreversible capacity loss of amorphous carbon in the first cycle. Thereafter, the Coulombic Efficiencies of both samples are well-preserved at about $100 \%$ at all current densities. This demonstrates that introduction of $\mathrm{N}$-doped amorphous carbon nanoarrays did not degrade the reversibility of the graphene nanoplatelets.

To investigate the origin of the improved capacity in GNC as compared to that of graphene nanoplatelets, typical discharge and charge profiles of G and GNC electrodes measured at different current densities are shown in Figures 4C,D, respectively. As seen from the discharge/charge curves in Figure 4C, the capacity of the G electrode is mainly contributed by $\mathrm{Li}^{+}$storage in the graphite lattice, which is represented by the flat voltage plateau below a certain value, such as $0.25 \mathrm{~V}$ at $0.1 \mathrm{~A}$ $\mathrm{g}^{-1}$. This Li storage behavior is very similar to that of graphite. The discharge/charge profiles of the GNC electrode show a short voltage plateau and a long sloped region, obviously different from that of graphene nanoplatelets. Such discharge/charge profiles can also be treated as overlap of the discharge/charge curves of the graphene nanoplatelets and the $\mathrm{N}$-doped amorphous carbon nanoarrays. In other words, the Li storage capacity in GNC is contributed by both components. Moreover, as seen in Figure $4 \mathrm{D}$, the voltage plateau associated to Li storage in graphite lattice almost disappears at 2 and $3 \mathrm{~A} \mathrm{~g}^{-1}$. The excellent high rate capacities of GNC anode are almost completely contributed by the sloped region, i.e., by the $\mathrm{N}$-doped amorphous carbon nanoarrays. This convincingly proves that anchoring vertical $\mathrm{N}$ doped amorphous carbon nanoarrays on both sides of graphene nanoplatelets has greatly improved the specific capacity and rate performance of the latter. Such excellent rate performance enables the hybrid material to be a potential anode of high power LIBs.

Figure 5 shows the cycle performances of the graphene nanoplatelets and GNC measured at $0.5 \mathrm{~A} \mathrm{~g}^{-1}$. It should be pointed out that the vibration of the cycling curves was due to the temperature fluctuation during daylight and night time. It is interesting to note that the specific capacity of the graphene nanoplatelets keeps increasing at the beginning 80 cycles and then maintains at about $260-290 \mathrm{~mA} \mathrm{~h} \mathrm{~g}^{-1}$, whereas that of GNC keeps increasing from 420 to about $540 \mathrm{~mA} \mathrm{~h} \mathrm{~g}^{-1}$ after 300 cycles. The capacity increase of both samples along cycling might be ascribed to gradual activation of the active materials. The Coulombic Efficiencies of both cells are nearly 100\%. As reported in our previous work (Jiang et al., 2017), the capacity of N-doped carbon anode derived from PPy would decrease gradually along cycling. The excellent cycle performance of 

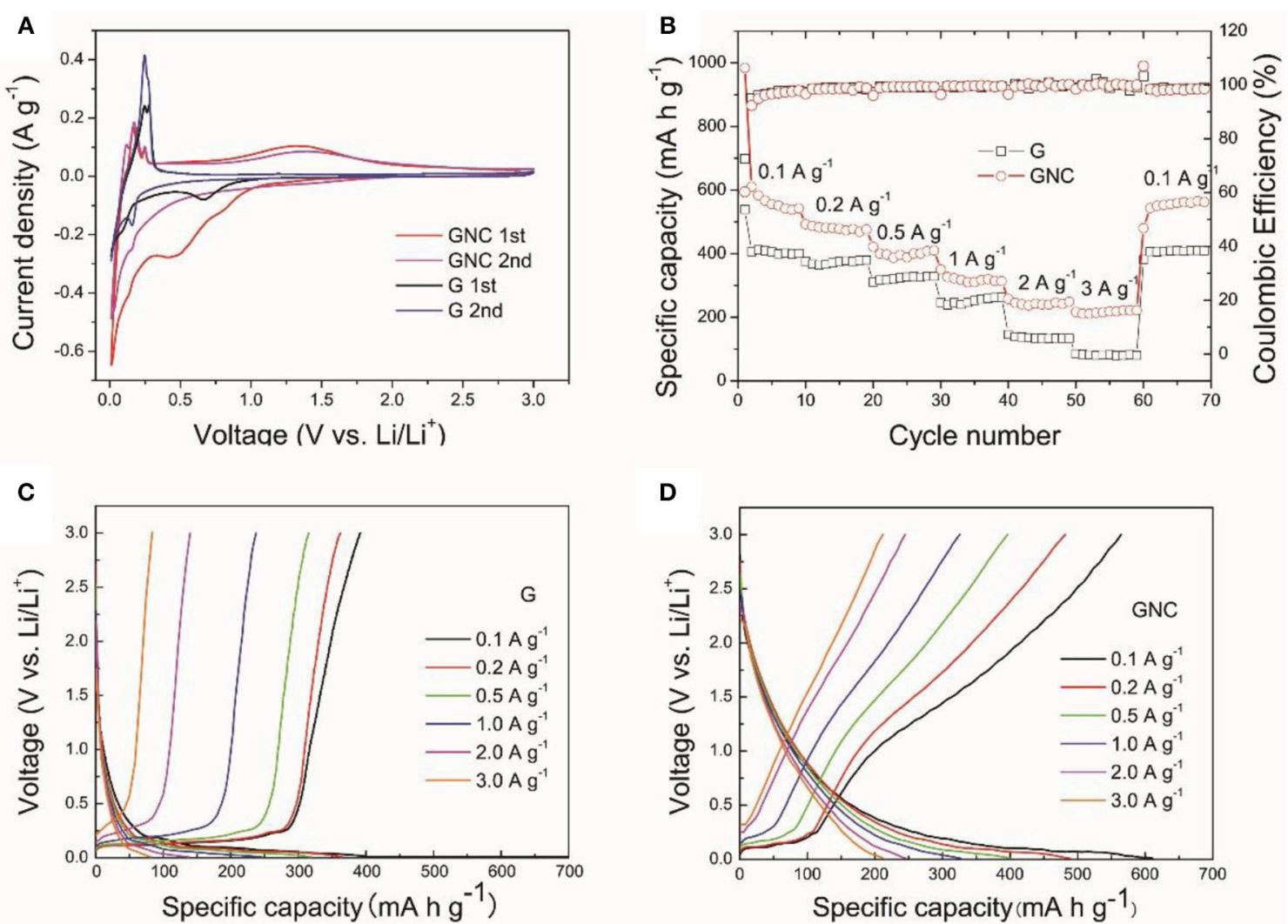

FIGURE 4 | The CV curves measured at a scan rate of $0.1 \mathrm{mV} \mathrm{s}^{-1} \mathbf{( A )}$, the rate capability (B), the discharge/charge profiles of the G (C), and GNC (D) electrodes at different current densities.

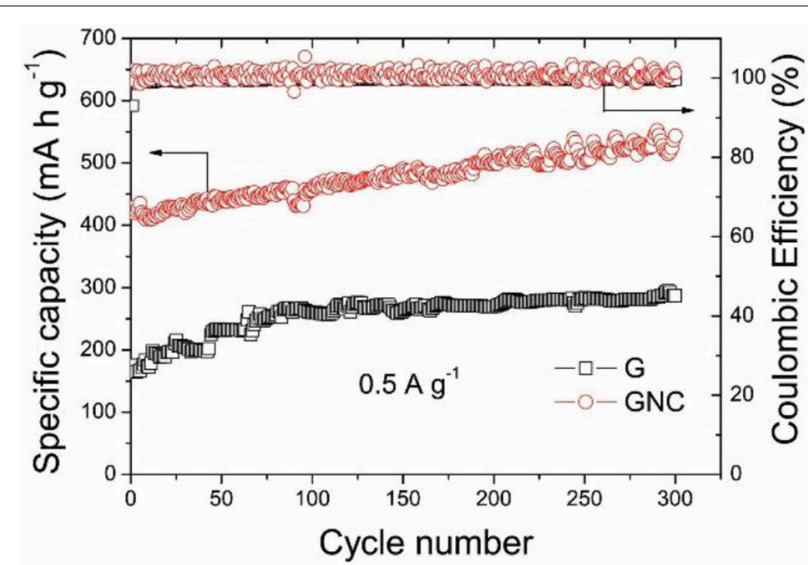

FIGURE 5 | Cycle performances of the graphene nanoplatelets and GNC at $0.5 \mathrm{~A} \mathrm{~g}^{-1}$.

GNC strongly suggests that the inner graphene nanoplatelets in the sandwich-structured GNC have stabilized the hybrid anode material during the cycling, whereas the vertical $\mathrm{N}$-doped amorphous carbon nanoarrays have provided high capacity and excellent rate performance. These synergistic effects have resulted in the outstanding electrochemical Li storage property of the GNC hybrid anode material.

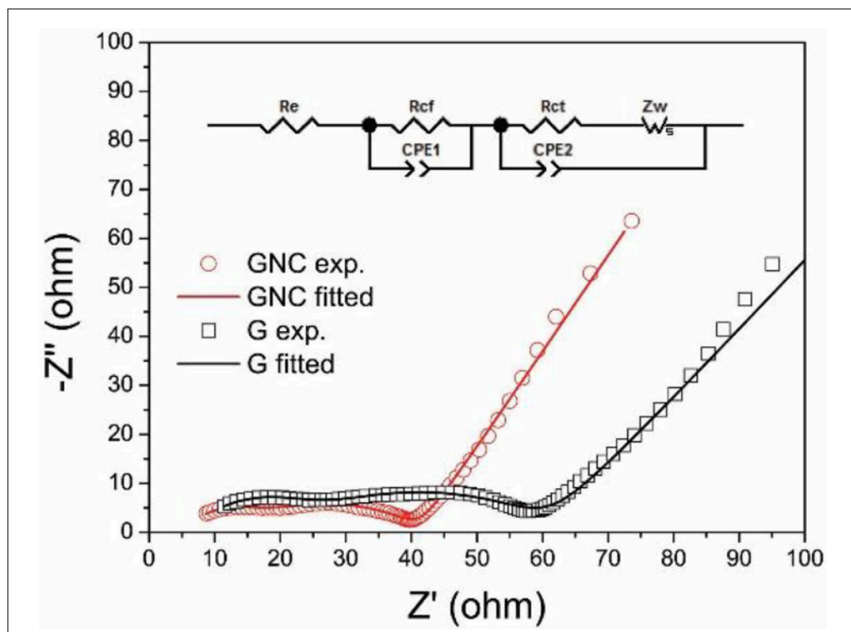

FIGURE 6 | The Nyquist plots of the half cells made from G and GNC.

The electrochemical impedance spectra (EIS) of the G//Li and $\mathrm{GNC} / / \mathrm{Li}$ half cells after the cycling tests were measured at the discharged state (about $0.01 \mathrm{~V}$ ). The Nyquist plots and the equivalent circuit used for the data fitting are shown in Figure 6. Both Nyquist plots show two compressed semicircles in the high and middle-frequency regions, which correspond to the resistance of $\mathrm{Li}^{+}$ions penetration through the SEI film $\left(R_{\mathrm{cf}}\right)$ 
and the charge transfer resistance between the electrolyte and electrode $\left(R_{\mathrm{ct}}\right)$, respectively. As fitted by Zview software using eh equivalent circuit shown in Figure 6 inset, the $R_{\mathrm{cf}}$ and $R_{\mathrm{ct}}$ values for GNC are 9.75 and $26.62 \Omega$, respectively, which are much smaller than those of the starting graphene nanoplatelets ( 16.03 and $39.05 \Omega$ ). This clearly indicates that the hybrid anode shows greatly reduced resistance of $\mathrm{Li}^{+}$ions transportation through the SEI film and the electrolyte/electrode interface, possibly due to the high electronic conductivity and the ultrathin sheet-like structure of the $\mathrm{N}$-doped amorphous carbon nanoarrays. The reduced charge transfer resistance may also account for the much improved rate capability of the GNC electrode.

\section{CONCLUSION}

Graphene itself is not an ideal anode material of Li-ion batteries due to its low packing density and severe aggregation of the ultrathin graphene sheets. In this work, a sandwich-structured hybrid anode was prepared by vertically anchoring nitrogendoped amorphous carbon nanoarrays on both sides of graphene nanoplatelets. The hybrid anode exhibits high capacity and excellent rate capability as well as stable cycle stability toward Li storage. High average discharge capacities of 562 and $217 \mathrm{~mA} \mathrm{~h}$ $\mathrm{g}^{-1}$ were achieved at 0.1 and $3 \mathrm{~A} \mathrm{~g}^{-1}$, which were much higher

\section{REFERENCES}

Assresahegn, B. D., Ossonon, B. D., and Bélanger, D. (2018). Graphene nanosheets and polyacrylic acid grafted silicon composite anode for lithium ion batteries. J. Power Sources 391, 41-50. doi: 10.1016/j.jpowsour.2018.03.067

Che, Z., Li, Y., Chen, K., and Wei, M. (2016). Hierarchical MoS2@RGO nanosheets for high performance sodium storage. J. Power Sources 331, 50-57. doi: 10.1016/j.jpowsour.2016.08.139

Chen, Z., Liu, Y., Zhang, Y., Shen, F., Yang, G., Wang, L., et al. (2018). Ultrafine layered graphite as an anode material for lithium ion batteries. Mater. Lett. 229, 134-137. doi: 10.1016/j.matlet.2018.06.104

Dong, Y., Wu, Z.-S., Ren, W., Cheng, H.-M., and Bao, X. (2017). Graphene: a promising $2 \mathrm{D}$ material for electrochemical energy storage. Sci. Bull. 62, 724-740. doi: 10.1016/j.scib.2017.04.010

Dutta, S., Bhaumik, A., and Wu, K. C. W. (2014). Hierarchically porous carbon derived from polymers and biomass: effect of interconnected pores on energy applications. Energy Environ. Sci. 7, 3574-3592. doi: 10.1039/C4EE01075B

Fujimoto, H. (2010). Development of efficient carbon anode material for a highpower and long-life lithium ion battery. J. Power Sources 195, 5019-5024. doi: 10.1016/j.jpowsour.2010.02.005

Hong, Z., Zhou, K., Zhang, J., Huang, Z., and Wei, M. (2016). Selfassembled synthesis of mesocrystalline $\mathrm{TiO}_{2} @ \mathrm{C}-\mathrm{rGO}$ hybrid nanostructures for highly reversible sodium storage. Crystal Growth Design 16, 6605-6612. doi: 10.1021/acs.cgd.6b01293

Jiang, C., Wang, J., Chen, Z., Yu, Z., Lin, Z., and Zou, Z. (2017). Nitrogendoped hierarchical carbon spheres derived from $\mathrm{MnO}_{2}$-templated spherical polypyrrole as excellent high rate anode of Li-ion batteries. Electrochim. Acta 245, 279-286. doi: 10.1016/j.electacta.2017.05.157

Jiang, Y., Yan, X., Mei, P., Zhang, Y., Xiao, W., and Tang, H. (2018). Electrochemical reconstruction induced high electrochemical performance of $\mathrm{Co}_{3} \mathrm{O}_{4} /$ reduced graphene oxide for lithium ion batteries. J. Alloys Compd. 764, 80-87. doi: 10.1016/j.jallcom.2018.06.044

Li, J., Yao, R., Bai, J., and Cao, C. (2013). Two-dimensional mesoporous carbon nanosheets as a high-performance anode material for lithium-ion batteries. ChemPlusChem 78, 797-800. doi: 10.1002/cplu.201300158 than the 404 and $81 \mathrm{~mA} \mathrm{~h} \mathrm{~g}^{-1}$ of the graphene nanoplatelets. The hybrid anode maintained a discharge capacity of $540 \mathrm{~mA} \mathrm{~h}$ $\mathrm{g}^{-1}$ after 300 cycles at $0.5 \mathrm{~A} \mathrm{~g}^{-1}$, demonstrating an outstanding cycle performance. It was believed that the vertically aligned highly conductive $\mathrm{N}$-doped amorphous carbon nanaoarrays have provided high capacity and excellent rate performance, and the inner graphene nanoplatelets have improved the cycle stability of the hybrid anode material. The results reported here may provide a versatile way to fabricate sandwich-structured hybrid material with $\mathrm{N}$-doped carbon nanoarrays vertically growing on a twodimensional substrate, hence building up a three-dimensional porous network for enhanced energy storage capacity.

\section{AUTHOR CONTRIBUTIONS}

ZZ and CJ proposed the work, performed materials characterizations and wrote the manuscript. LW and JC fabricated the materials and performed LIB testing.

\section{FUNDING}

This work is supported by Natural Science Foundation (No. 2016J01746) and Guidance Project (No. 2016H0038), Minjiang Scholarship program (No. 60815002) of Fujian Province, China.

Liu, C., Liu, X., Tan, J., Wang, Q., Wen, H., and Zhang, C. (2017). Nitrogendoped graphene by all-solid-state ball-milling graphite with urea as a high-power lithium ion battery anode. J. Power Sources 342, 157-164. doi: 10.1016/j.jpowsour.2016.11.110

Liu, H., Jia, M., Cao, B., Chen, R., Lv, X., Tang, R., et al. (2016). Nitrogen-doped carbon/graphene hybrid anode material for sodium-ion batteries with excellent rate capability. J. Power Sources 319, 195-201. doi: 10.1016/j.jpowsour.2016.04.040

Liu, X., Li, Z., Fei, H., and Wei, M. (2015). Composite of K-doped $\left(\mathrm{NH}_{4}\right)_{2} \mathrm{~V}_{3} \mathrm{O}_{8}$ /graphene as an anode material for sodium-ion batteries. Dalton Trans. 44, 18864-18869. doi: 10.1039/C5DT0 3239C

Nitta, N., Wu, F., Lee, J. T., and Yushin, G. (2015). Li-ion battery materials: present and future. Mater. Today 18, 252-264. doi: 10.1016/j.mattod.2014.1 0.040

Qie, L., Chen, W. M., Wang, Z. H., Shao, Q. G., Li, X., Yuan, L. X., et al. (2012). Nitrogen-doped porous carbon nanofiber webs as anodes for lithium ion batteries with a superhigh capacity and rate capability. Adv. Mater. 24, 2047-2050. doi: 10.1002/adma.201104634

Qiu, H., Zeng, L., Lan, T., Ding, X., and Wei, M. (2015). In situ synthesis of $\mathrm{GeO}_{2}$ /reduced graphene oxide composite on Ni foam substrate as a binder-free anode for high-capacity lithium-ion batteries. J. Mater. Chem. A 3, 1619-1623. doi: 10.1039/C4TA05212A

Selvamani, V., Ravikumar, R., Suryanarayanan, V., Velayutham, D., and Gopukumar, S. (2016). Garlic peel derived high capacity hierarchical N-doped porous carbon anode for sodium/lithium ion cell. Electrochim. Acta 190, 337-345. doi: 10.1016/j.electacta.2016.01.006

Shi, S., Hua, X., and Guo, H. (2018). Microwave-assisted one-pot synthesis of $\mathrm{SnC}_{2} \mathrm{O}_{4}$ /graphene composite anode material for lithium-ion batteries. Ceram. Int. 44, 13495-13501. doi: 10.1016/j.ceramint.2018.0 4.179

Striebel, K. A., Shim, J., Cairns, E. J., Kostecki, R., Lee, Y.-J., Reimer, J., et al. (2004). Diagnostic analysis of electrodes from high-power lithium-ion cells cycled under different conditions. J. Electrochem. Soc. 151, A857-A866. doi: $10.1149 / 1.1710514$ 
Tong, X., Wang, H., Wang, G., Wan, L., Ren, Z., Bai, J., et al. (2011). Controllable synthesis of graphene sheets with different numbers of layers and effect of the number of graphene layers on the specific capacity of anode material in lithiumion batteries. J. Solid State Chem. 184, 982-989. doi: 10.1016/j.jssc.2011.0 3.004

Wang, H., and Yoshio, M. (2001). Carbon-coated natural graphite prepared by thermal vapor decomposition process, a candidate anode material for lithiumion battery. J. Power Sources 93, 123-129. doi: 10.1016/S0378-7753(00)00552-8

Wang, J., Lin, L., and He, D. (2018). Self-assembly of $\mathrm{Fe}_{2} \mathrm{O}_{3}$ nanotubes on graphene as an anode material for lithium ion batteries. J. Alloys Compd. 750, 871-877. doi: 10.1016/j.jallcom.2018.04.079

Wang, R., Wang, S., Jin, D., Zhang, Y., Cai, Y., Ma, J., et al. (2017). Engineering layer structure of MoS2-graphene composites with robust and fast lithium storage for high-performance Li-ion capacitors. Energy Storage Mater. 9, 195-205. doi: 10.1016/j.ensm.2017.07.013

Wu, Y.-P., Wan, C.-R., Jiang, C.-Y., Fang, S.-B., and Jiang, Y.-Y. (1999). Mechanism of lithium storage in low temperature carbon. Carbon 37, 1901-1908. doi: 10.1016/S0008-6223(99)00067-6

Xu, J., Han, Z., Wu, J., Song, K., Wu, J., Gao, H., et al. (2018). Synthesis and electrochemical performance of vertical carbon nanotubes on few-layer graphene as an anode material for Li-ion batteries. Mater. Chem. Phys. 205, 359-365. doi: 10.1016/j.matchemphys.2017.11.039
Zhang, L., Zhang, M., Wang, Y., Zhang, Z., Kan, G., Wang, C., et al. (2014). Graphitized porous carbon microspheres assembled with carbon black nanoparticles as improved anode materials in Li-ion batteries. J. Mater. Chem. A 2, 10161-10168. doi: 10.1039/C4TA00356J

Zhang, W., Xu, T., Liu, Z., Wu, N. L., and Wei, M. (2018). Hierarchical $\mathrm{TiO}_{2}-\mathrm{x}$ imbedded with graphene quantum dots for high-performance lithium storage. Chem. Commun. 54, 1413-1416. doi: 10.1039/C7CC09406J

Zhou, D., Liu, Y., Song, W.-L., Li, X., Fan, L.-Z., and Deng, Y. (2017). Three-dimensional porous carbon-coated graphene composite as high-stable and long-life anode for sodium-ion batteries. Chem. Eng. J. 316, 645-654. doi: 10.1016/j.cej.2017.02.008

Conflict of Interest Statement: The authors declare that the research was conducted in the absence of any commercial or financial relationships that could be construed as a potential conflict of interest.

Copyright (C) $2018 \mathrm{Zou}, \mathrm{Wu}$, Chen and Jiang. This is an open-access article distributed under the terms of the Creative Commons Attribution License (CC BY). The use, distribution or reproduction in other forums is permitted, provided the original author(s) and the copyright owner(s) are credited and that the original publication in this journal is cited, in accordance with accepted academic practice. No use, distribution or reproduction is permitted which does not comply with these terms. 